\subsection{GONOCOCCAL PHARYNGEAL SUSCEPTIBILITY TO CEFTRIAXONE, CEFIXIME AND AZITHROMYCIN AMONG MEN AND WOMEN IN EGISP, 2018 - 2020}

S St Cyr ${ }^{*}$, C Pham, E Torrone, H Weinstock, eGISPWorking Group. Division of STD Prevention, Centers for Disease Control and Prevention, Atlanta, USA

\subsection{6/sextrans-2021-sti.100}

Background Most gonorrhea treatment failures on ceftriaxonebased regimens, the foundation of current treatment recommendations, have been associated with pharyngeal infections; however, antimicrobial susceptibility data for pharyngeal infections are limited. We present preliminary pharyngeal susceptibility data collected through a U.S. sentinel surveillance project.

Methods The enhanced Gonococcal Isolate Surveillance Project (eGISP) collects gonococcal isolates from male and female genital and extragenital sites. Samples are collected for culture from symptomatic and/or exposed patients at participating STD clinics. Gonococcal isolates undergo antimicrobial susceptibility testing by agar dilution with results reported as minimum inhibitory concentrations (MIC). Since eGISP began, 13 clinical sites across the U.S. have participated in eGISP.

Results During 2018-2020, 628 pharyngeal isolates were collected in eGISP among men who have sex with men (MSM) (n=370; 59\%), men who have sex with women only (MSW) $(n=142 ; 23 \%)$ and women $(n=116 ; 18 \%)$. Two isolates displayed reduced susceptibility to ceftriaxone (MIC $\geq 0.125 \mu \mathrm{g} / \mathrm{mL}$ ), an overall point prevalence of $0.3 \%$; both isolates were among MSM. One isolate in an MSM displayed reduced susceptibility to cefixime (MIC $\geq 0.25 \mu \mathrm{g}$ / $\mathrm{mL}$ ), a prevalence of $0.2 \%$ overall. The prevalence of reduced susceptibility to azithromycin (MIC $\geq 2.0 \mu \mathrm{g} / \mathrm{mL}$ ) was $8.0 \%$ overall (8.4\% MSM, 8.5\% MSW, 6.0\% women). Similar patterns were seen among the 3898 urogenital isolates collected; overall prevalence of reduced susceptibility for ceftriaxone was $0.1 \% \quad(0 \%$ MSM, $0.1 \%$ MSW, $0.4 \%$ women), for cefixime was $0.3 \%$ (0.4\% MSM, $0.3 \%$ MSW, $0 \%$ women), and for azithromycin was $6.4 \%$ (8.8\% MSM, 5.3\% MSW, 3.4\% women).

Conclusion Based on sentinel surveillance in multiple U.S. cities, the prevalence of pharyngeal reduced susceptibility to cephalosporins appears low; however, prevalence of reduced susceptibility to azithromycin was high among MSM, MSW, and women. As many pharyngeal gonococcal infections are asymptomatic, monitoring susceptibility patterns at this anatomic site can support public health action to ensure effective treatment.

\subsection{CHANGES IN GONOCOCCAL INCIDENCE, SCREENING, AND REPORTING AS EXPLANATIONS FOR INCREASED GONOCOCCAL CASE REPORTS BETWEEN 2008 AND 2018, UNITED STATES}

I Spicknall*. Centers For Disease Control and Prevention, USA

\subsection{6/sextrans-2021-sti.101}

Background Though gonococcal case reports are related to incidence, case report increases may not necessarily indicate incidence increases. This results from incidence also being related to testing, reporting, and gonococcal natural history.
In this work, we estimate the levels of change necessary in gonococcal incidence, testing, and reporting to explain changes in case reports between 2008 and 2018 in the United States.

Methods We applied a recently published mathematical model that relates gonococcal incidence to natural history (natural clearance rate, symptomatic probability), testing (background screening and symptomatic treatment seeking), and reporting (percent of diagnosed infections reported to CDC). We assumed natural history parameters did not change between 2008-2018. Using the closed form mathematical modeling solution for incidence, we determined what change in incidence, testing, and reporting was necessary to explain the observed changes in case reports.

Results Assuming no changes in the determinants of incidence between 2008-2018, 2018 estimated incidence was 1.2-3 times higher than 2008 incidence (depending on age and sex), except in 15-19-year-old women, where it was $15 \%$ higher in 2008. Changes in background screening could explain the increases if 2008 screening in women was substantially lower (8-20\% of 2018 values, depending on age); in men, even with zero assumed 2008 screening, this alone was unable explain these increases. For symptomatic treatment seeking, 2008 levels would need to be 5-60\% of 2018 values, depending on age and sex. For case reporting, 2008 levels would need to be $30-85 \%$ of 2018 values.

Conclusions Between 2008-2018, assuming no changes in testing or reporting, estimated gonococcal incidence increased, most apparently in 25-39-year-olds. Changes in testing and reporting necessary to explain these increases seem unrealistic (e.g., less than half of 2008 diagnosed gonorrhea being reported). Thus, these increased estimates likely reflect some true increase in gonococcal incidence, particularly in older age groups.

\subsection{CHARACTERISTICS OF PEOPLE WITH GONORRHEA WITH REDUCED AZITHROMYCIN ANTIMICROBIAL SUSCEPTIBILITY IN 8 JURISDICTIONS IN THE UNITED STATES, 2018-2019}

${ }^{1} \mathrm{E}$ Learner ${ }^{*},{ }^{2} \mathrm{~K}$ Gieseker, ${ }^{1} \mathrm{~K}$ Mauk, ${ }^{1} \mathrm{~K}$ Town, ${ }^{1} \mathrm{C}$ Pham, ${ }^{1} \mathrm{~S}$ St Cyr, ${ }^{3} \mathrm{D}$ Shrestha, ${ }^{4} \mathrm{~L}$ Barbee, ${ }^{5} \mathrm{C}$ McNeil, ${ }^{1,6}$ J Holderman, ${ }^{7} \mathrm{~K}$ Johnson, ${ }^{8} \mathrm{~T}$ Nguyen, ${ }^{9} \mathrm{~T}$ Albano, ${ }^{1} \mathrm{~K}$ Schlanger, ${ }^{1} \mathrm{R}$ Kirkcaldy. ${ }^{1}$ Centers For Disease Control and Prevention; ${ }^{2}$ Colorado Department of Public Health and Environment; ${ }^{3}$ Wisconsin Department of Health; ${ }^{4}$ Public Health - Seattle and King County; ${ }^{5}$ Guilford County Department of Health; ${ }^{6}$ Indiana State Department of Health; ${ }^{7}$ New York City Department of Health and Mental Hygiene; ${ }^{8}$ San Francisco Department of Public Health; ${ }^{9}$ Hawaii State Department of Health, United States

\subsection{6/sextrans-2021-sti.102}

Background In the USA, reduced azithromycin susceptibility (AZM-RS) gonococci have been observed in men who have sex with men (MSM), women, and men who have sex with women only (MSW). However AZM-RS prevalence and epidemiological characteristics among women and MSW are not well-described. We examined epidemiological characteristics of MSM and a combined group of women and MSW with susceptible and AZM-RS gonorrhea to investigate differences between groups.

Methods We analyzed epidemiological and susceptibility data from people attending STD and community clinics in eight jurisdictions participating in CDC's Strengthening U.S. Response to Resistant Gonorrhea project during 2018-2019. 
We defined people with AZM-RS gonorrhea as those with $\geq 1$ gonococcal isolate demonstrating a minimum inhibitory concentration of $\mathrm{AZM} \geq 2.0 \mu \mathrm{g} / \mathrm{mL}$. We used inverse variance weighting to account for heterogeneity in sample size across jurisdictions to estimate pooled AZM-RS prevalences and 95\% confidence intervals.

Results Across eight sites, 8,859 people (4,521 MSM, 758 women, and 3,580 MSW) provided at least one isolate for susceptibility testing; 1,052 people (10.4\% [95\% CI: 6.4\%14.4\%]) had gonorrhea demonstrating AZM-RS. AZM-RS prevalence was markedly high among MSM (15.1\% [95\% CI: 10.2\%-20.0\%]), and lower yet elevated among women and MSW combined (5.3\% [95\% CI: 2.9\%-7.7\%]). Among MSM with AZM-RS gonorrhea, 16.2\% (95\% CI: 10.9\%-21.4\%) reported having $3+$ sexual partners in the last 2-3 months and $16.7 \%$ (95\% CI: $12.6 \%-20.9 \%$ ) reported previous gonococcal infections. Among women/MSW with AZM-RS, 6.2\% (95\% CI: $3.7 \%-8.7 \%)$ reported $3+$ recent sexual partners, and $4.8 \%$ (95\% CI: $2.4 \%-7.3 \%)$ reported previous gonococcal infections.

Conclusions AZM-RS prevalence among women/MSW was lower than among MSM but still elevated, and a lower proportion of women/MSW reported multiple recent sexual partners and previous gonococcal infections. These data highlight differences in the epidemiology of reduced gonococcal susceptibility and the need to tailor resistant gonorrhea control approaches to affected populations.

\subsection{TYPING OUTER MEMBRANE VESICLE PROTEINS OF NEISSERIA GONORRHOEAE PROVIDES INSIGHT INTO ANTIMICROBIAL RESISTANCE}

${ }^{1} \mathrm{~A}$ Unitt*, ${ }^{1} \mathrm{C}$ Rodrigues, ${ }^{1} \mathrm{~J}$ Bray, ${ }^{1} \mathrm{~K}$ Jolley, ${ }^{2} \mathrm{C}$ Tang, ${ }^{1} \mathrm{M}$ Maiden, ${ }^{1} \mathrm{O}$ Harrison. ${ }^{1}$ The Maiden Lab, University of Oxford, Oxford, UK; ${ }^{2}$ The Tang Lab, University of Oxford, Oxford, UK

10.1136/sextrans-2021-sti.103

Background Outer membrane vesicles (OMVs) are known to be produced in significant quantities during gonococcal infection, however, their role remains mostly unknown. In this study, the diversity of gonococcal OMV proteins and their association with antimicrobial resistance (AMR) was investigated using computational methods.

Methods Omics-based approaches were employed through the PubMLST.org/neisseria database to analyse a selection of 26 proteins, including 23 identified in $\mathrm{N}$. gonorrhoeae OMVs by Deo et al.(2018). These proteins were annotated across 4884 isolates from 58 countries and formed into an OMV peptide typing scheme. This facilitated the cataloguing of OMV protein diversity across the gonococcal population by the identification of OMV sequence types (OMV STs). The association of OMV STs with sequence typing schemes that categorise the core genome ( $\left.\mathrm{Ng} \mathrm{cgc}_{-} 400\right)$ and AMR (NG STAR) was then assessed, primarily using the Cramer's V statistic.

Results 2120 unique gonococcal OMV STs were identified. High levels of association were found between these OMV STs and both the core genome (OMV ST vs $\mathrm{Ng}$ cgc 400: Cramer's V $=1.00$ ) and AMR (OMV ST vs NG STAR ST: Cramer's $\mathrm{V}=0.967)$. This is consistent the potential involvement of OMVs in the generation of AMR in N. gonorrhoeae. Conclusion These results suggest that OMVs are more significantly involved in antimicrobial resistance in $\mathrm{N}$. gonorrhoeae than previously thought, providing a new avenue for research that could inform future efforts to limit AMR evolution and treat resistant infections. Additionally, the study demonstrates the role that population-level Omics-based approaches can play in improving our understanding of sexually transmitted infections. This applies not only to AMR, but also to other traits such as transmissibility or virulence.

\section{Chlamydia epidemiology}

\subsection{INCIDENT UROGENITAL AND ANORECTAL CHLAMYDIA TRACHOMATIS IN WOMEN: THE ROLE OF SEXUAL EXPOSURE AND AUTO-INOCULATION; A MULTICENTRE OBSERVATIONAL STUDY (FEMCURE)}

\begin{abstract}
${ }^{1,2} \mathrm{~N}$ Dukers-Muijrers* ${ }^{*},{ }^{3,4} \mathrm{M}$ Schim van der Loeff, ${ }^{5} \mathrm{P}$ Wolffs, ${ }^{3,6}$ S Bruisten, ${ }^{7,8,9} \mathrm{H}$ Götz, ${ }^{3} \mathrm{~T}$ Heijman, ${ }^{3,6} \mathrm{H}$ Zondag, ${ }^{5} \mathrm{M}$ Lucchesi, ${ }^{3,9,10} \mathrm{H}$ de Vries, ${ }^{1,5,11} \mathrm{C}$ Hoebe. ${ }^{1}$ Department of Sexual Health, Infectious Diseases, and Environmental Health, South Limburg Public Health Service, Heerlen, The Netherlands; ${ }^{2}$ Department of Health Promotion, Care and Public Health Research Institute (CAPHRI), Maastricht University Medical Center (MUMC+), Maastricht, The Netherlands; ${ }^{3}$ Department of Infectious Diseases, Public Health Service of Amsterdam (GGD Amsterdam), Amsterdam, The Netherlands; ${ }^{4}$ Department of Internal Medicine, Amsterdam Infection and Immunity Institute (All), Amsterdam University Medical Center (UMC), Amsterdam, The Netherlands; ${ }^{5}$ Department of Medical Microbiology, Care and Public Health Research Institute (CAPHRI), Maastricht University Medical Center (MUMC+), Maastricht, The Netherlands; ${ }^{6}$ Department of Medical Microbiology, Amsterdam Infection and Immunity Institute (All), Amsterdam University Medical Center (UMC), Amsterdam, The Netherlands; 'Department of Public Health, Erasmus MC - University Medical Center Rotterdam, Rotterdam, The Netherlands; ${ }^{8}$ Department Infectious Disease Control, Municipal Public Health Service Rotterdam-Rijnmond (GGD Rotterdam-Rijnmond), Rotterdam, The Netherlands; ${ }^{9}$ National Institute of Public Health and the Environment (RIVM), Epidemiology and Surveillance Unit, Centre for Infectious Disease Control, Bilthoven, The Netherlands; ${ }^{10}$ Amsterdam UMC, University of Amsterdam, department of dermatology, Amsterdam Institute for Infection and Immunity (AlI), location Academic Medical Centre, Amsterdam, The Netherlands; ${ }^{11}$ Department of Social Medicine, Care and Public Health Research Institute (CAPHRI), Maastricht University Medical Center (MUMC+), Maastricht, The Netherlands
\end{abstract}

\subsection{6/sextrans-2021-sti.104}

Background Anorectal infections with Chlamydia trachomatis (CT) are prevalent in women visiting STI-clinics. In women, azithromycin treatment in anorectal chlamydia is unsuccessful in about 20\%, with the potential of subsequent re-infection of the vagina through autoinoculation. We evaluated the risk for incident urogenital CT, by exposure from the own anorectal site and exposure by sex; and similarly evaluated risks for incident anorectal CT.

Methods Prospective multicenter cohort study, FemCure. At 4, $6,8,10$, and 12 weeks after azithromycin or doxycycline CT treatment, women self-collected anorectal and urogenital swabs for CT-DNA testing. We calculated the proportion with incident CT, at week 6-12, by 2 -week time-periods. Compared to no exposure (A), risk of incident urogenital CT was estimated for sexual exposure (B), anorectal CT exposure (C), and both exposures (D), adjusted for confounders by adjusted odds ratios (OR) and 95\% confidence intervals (CI). We similarly assessed incident anorectal CT.

Results Data comprised 385 participants contributing 1540 two-week periods.

Urogenital incidence was $3 \cdot 3 \% \quad(47 / 1428)$ [95\%CI: $2 \cdot 4$ 4.4]; $0.7 \%$ (A), $1.9 \%$ (B), $13.9 \%$ (C), and $25 \cdot 4$ (D). ORs were: $2 \cdot 7$ [95\%CI:0.9-8.6] (B), $21 \cdot 8$ [95\%CI:6.7-70.7] (C), $49 \cdot 7$ [95\%CI:15.4-160.4] (D).

Anorectal incidence was $2 \cdot 9 \% \quad(39 / 1343)$ [95CI:1.8-3.6]; $1 \cdot 3 \%(\mathrm{~A}), 1 \cdot 3 \%(\mathrm{~B}), 27 \cdot 8 \%(\mathrm{C})$, and $36 \cdot 7 \%(\mathrm{D})$. ORs were: 